\title{
Improvement in exercise capacity and delayed anaerobic metabolism induced by far-infrared-emitting garments in active healthy subjects: A pilot study
}

European Journal of Preventive Cardiology $0(00)$ I-8

(C) The European Society of Cardiology 2018

Reprints and permissions: sagepub.co.uk/journalsPermissions.nav DOI: 10.1 I77/20474873/8768598 journals.sagepub.com/home/ejpc

(SAGE

\author{
Valentina Mantegazza', Mauro Contini', Maurizia Botti' \\ Ada Ferri ${ }^{3}$, Francesca Dotti ${ }^{3}$, Pierluigi Berardi ${ }^{4}$ and \\ Piergiuseppe Agostoni ${ }^{5}$
}

\begin{abstract}
Background: Far-infrared-emitting garments have several biological properties including the capability to increase blood perfusion in irradiated tissues.

Design: The aim of the study was to evaluate whether far-infrared radiation increases exercise capacity and delays anaerobic metabolism in healthy subjects.

Methods: With a double-blind, crossover protocol, a maximal cardiopulmonary exercise test was performed in 20 volunteers, wearing far-infrared or common sport clothes, identical in texture and colour.

Results: Comparing far-infrared with placebo garments, higher oxygen uptake at peak of exercise and longer endurance time were observed (peak oxygen uptake $38.0 \pm 8.9$ vs. $36.2 \pm 8.5 \mathrm{ml} / \mathrm{kg} / \mathrm{min}$, endurance time $592 \pm 85$ vs. $570 \pm 7 \mathrm{I}$ seconds; $P<0.01$ ); the anaerobic threshold was significantly delayed (anaerobic threshold time $46 \mathrm{I} \pm 93$ vs. $4 \mathrm{I} 7 \pm \mathrm{I} 03$ seconds) and anaerobic threshold oxygen uptake and anaerobic threshold oxygen pulse were significantly higher $(25.3 \pm 6.4$ vs. $20.9 \pm 5.4 \mathrm{ml} / \mathrm{kg} / \mathrm{min}$ and $13.3 \pm 3.8 \mathrm{vs}$. $12.4 \pm 3.3 \mathrm{ml} /$ beat, respectively). In 10 subjects the blood lactate concentration was measured every 2 minutes during exercise and at peak; lower values were observed with far-infrared fabrics compared to placebo from the eighth minute of exercise, reaching a significant difference at 10 minutes $(3.6 \pm 0.83$ vs. $4.4 \pm 0.96 \mathrm{mmol} / \mathrm{l} ; P=0.02)$.

Conclusions: In healthy subjects, exercising with a far-infrared outfit is associated with an improvement in exercise performance and a delay in anaerobic metabolism. In consideration of the acknowledged non-thermic properties of functionalised clothes, these effects could be mediated by an increase in oxygen peripheral delivery secondary to muscular vasodilation. These data suggest the need for testing far-infrared-emitting garments in patients with exercise limitation or in chronic cardiovascular and respiratory patients engaged in rehabilitation programmes.
\end{abstract}

\section{Keywords}

Far-infrared-emitting fabrics, muscular perfusion, peripheral oxygen delivery, exercise performance, cardiopulmonary exercise test, anaerobic metabolism, anaerobic threshold

Received 16 October 2017; accepted 13 March 2018

\section{Introduction}

Far-infrared radiation (FIR)-emitting fabrics are claimed to be biologically active textiles. ${ }^{1-3}$ Such garments are derived from traditional textile fibres by the incorporation of ceramic particles, which are natural minerals with peculiar electromagnetic absorption and emission properties. After absorbing incoming infrared radiation from the human body, FIR-emitting

\footnotetext{
'Heart Failure Unit, Centro Cardiologico Monzino IRCCS, Italy

${ }^{2}$ Engineering Department, Politecnico di Milano, Italy

${ }^{3}$ Engineering Department, Politecnico di Torino, Italy

${ }^{4}$ Marketing of $\mathrm{CNILIT}^{\circledR}$ Fiber Division, Italy

${ }^{5}$ Department of Clinical Sciences and Community Health, University of Milan, Italy
}

\section{Corresponding author:}

Piergiuseppe Agostoni, Cardiovascular Section, Department of Clinical Sciences and Community Health, Centro Cardiologico Monzino, University of Milan, Via Parea 4, 20138 Milan, Italy.

Email: piergiuseppe.agostoni@ccfm.it 
materials re-emit radiative energy at a longer wavelength between 4 and $16 \mu \mathrm{m}$, reflecting it back to the body. ${ }^{4,5}$ The emissivity of such engineered textile is a function of the percentage mass of bioceramics embedded into the fabric. ${ }^{6}$

In the band of infrared radiation, only FIR transfers energy in the form of gentle radiant heat with high penetrability in most biological material, up to $4 \mathrm{~cm}$ beneath the human skin, where it is perceived by local thermoreceptors. ${ }^{4,7}$ In human cells, there are charged groups located at specific molecule sites and associated with water molecules. The interaction between FIR and biological structures determines a vibrational and rotational stimulation of intra-molecular bonds, ${ }^{9}$ thus exerting an influence on the structure and behaviour of the whole molecule, which is proportionate to the water content. ${ }^{4}$ This phenomenon increases the molecular kinetic energy which finally results in local (skin and muscular) temperature rise, ${ }^{4,10}$ tissue vasodilation and therefore the enhancement of blood microcirculation in targeted regions of the body, such as exercising muscles. The same vasodilating effect is also induced by the nonthermic biological properties of FIR. ${ }^{1-4,7,8,11}$

By the Fick equation, oxygen consumption $\left(\mathrm{VO}_{2}\right)=$ cardiac output $*$ arteriovenous oxygen $\left(\mathrm{O}_{2}\right)$ difference $\left(\Delta \mathrm{a}-\mathrm{vO}_{2}\right){ }^{12}$ Consistently, enhanced muscular perfusion could increase peripheral oxygen delivery and thereby improve maximal $\mathrm{VO}_{2}$ and prolong aerobic metabolism. However, previous studies report contrasting effects of local vasodilation both on global exercise capacity and exercising muscle performance. ${ }^{13-16}$

Therefore, the aim of our study was twofold: (a) to evaluate the timing of anaerobic metabolism; and (b) to assess exercise capacity with a ramp incremental exercise in normal subjects wearing FIR-emitting tissues, as analysed by cardiopulmonary exercise test (CPET) ${ }^{17}$

\section{Methods}

This is a double-blind, randomised crossover, prospective study comparing the effects on exercise performance of two kinds of sport outfit in healthy volunteers, each of them serving as his or her own control. The outfit consisted of a T-shirt and shorts; one made of polyamide fabrics knitted with ceramic-bearing fibres, and one was the control counterpart, identical in colour, knit structure, texture, thickness and weight but without ceramic-bearing fibres.

As no data were available in the literature regarding the effects of FIR-emitting garments on exercise capacity, we could not establish an adequate sample size a priori and the present research must be considered within the frame of a pilot study.

Twenty healthy recreationally active subjects, already familiarised to CPET, were enrolled in the study and none of them withdrew from the study protocol. Ten volunteers were tested in an Italian research exercise laboratory and 10 were evaluated by $\mathbb{C N N I L I T}^{\circledR}$ Ltd., who guarantee the quality of the tests as well as the accuracy and truthfulness of the collected data. Similar equipment and protocol were used in both laboratories.

The study adhered to the ethical standards of the Declaration of Helsinki and its later amendments. All subjects signed an informed consent for the exercise procedure and scientific use of data.

Assignment to either outfit was designed in a random order by ${ }^{C}$ NILIT $^{\circledR}$ Ltd. and was concealed to researchers and volunteers. All subjects performed two maximal $\mathrm{CPET}^{18}$ on a cycle ergometer (Sensor Medics, Yorba Linda, CA, USA), in the same environmental conditions (laboratory temperature $22-23^{\circ} \mathrm{C}, \leq 50 \%$ relative humidity) and separated by one week, wearing either the functionalised or the non-functionalised outfit. Subjects were instructed not to modify their eating habits or training activity throughout the study protocol; in particular the day before the test, participants were asked not to ingest caffeine or alcohol and to refrain from strenuous exercise.

In particular, an individualised ramp protocol was applied, aimed at a test duration of $10 \pm 2$ minutes, ${ }^{19}$ with a progressive workload increase. Ventilation, expired and inspired gases were measured breath by breath $^{20}$ (V-max 229D Spectra metabolic cart; Sensor Medics, Yorba Linda, CA, USA) and collected data were analysed as a 20 seconds average. During the test, heart rate (HR), 12-lead ECG and pulse oxygen saturation (Radical 7, Masimo, Irvine, California, USA) were continuously monitored. ${ }^{20}$ Blood pressure was measured every 2 minutes using a cuff sphygmomanometer. ${ }^{20}$ Exercise was self-limited by the subjects after pushing themselves to exhaustion.

Among all data, oxygen uptake at peak of exercise (peak $\mathrm{VO}_{2}$ ) was measured at presumed maximal effort as the highest $\mathrm{VO}_{2}$ value, averaged over a 20 second period; ${ }^{21}$ it was considered correspondent to maximal $\mathrm{VO}_{2}$ if the following criteria were achieved: attainment of a plateau in $\mathrm{VO}_{2}$ graph in spite of incremental work rate, respiratory exchange ratio (RER) of 1.1 or greater and $\mathrm{HR}$ of $85 \%$ or greater of maximal predicted value. Peak $\mathrm{VO}_{2}$ was expressed both in absolute value $(\mathrm{ml} / \mathrm{kg} /$ $\mathrm{min}$ ) and as a percentage of maximal predicted value $\left(\mathrm{VO}_{2} \%\right)$, estimated for each subject according to the Wasserman-Hansen formula. ${ }^{22}$

Moreover, peak oxygen pulse $\left(\mathrm{O}_{2}\right.$ pulse $)$ was automatically provided by the software. It is the $\mathrm{VO}_{2} / \mathrm{HR}$ ratio at peak of exercise and reflects the amount of oxygen consumed per heart beat at the end of a maximal incremental exercise. ${ }^{22}$ 
Furthermore, the anaerobic threshold (AT) was determined using the $\mathrm{V}$-slope analysis from the plot of carbon dioxide production $\left(\mathrm{VCO}_{2}\right)$ versus $\mathrm{VO}_{2}$ on an equal scale s.24 $^{23}$ and confirmed by the consequent behaviour of carbon dioxide and oxygen end tidal pressures and ventilatory equivalents. ${ }^{25-27} \mathrm{O}_{2}$ pulse (AT $\mathrm{O}_{2}$ pulse), time at the AT (AT time) and timing of AT with respect to total endurance time (AT/ET ratio) were reported, as well as $\mathrm{VCO}_{2}\left(\mathrm{AT} \mathrm{VCO}_{2}\right)$, $\mathrm{HR}$ (AT HR) and oxygen uptake $\left(\mathrm{AT} \mathrm{VO}_{2}\right)$ expressed both as absolute values $(\mathrm{ml} / \mathrm{kg} / \mathrm{min})$ and as percentages of predicted maximal oxygen uptake $\left(\mathrm{AT} \mathrm{VO}_{2} \%\right)$.

In 10 volunteers (tested in the Italian research exercise laboratory), a blood sample from an antecubital vein was also drawn for blood lactate concentration (BLC) measurement at rest, every 2 minutes of loaded exercise and at peak.

Finally, a wireless sensor was fixed on volunteers' chests by means of a plaster to monitor the temperature of the microclimate between the skin and the T-shirt.

\section{Statistical analysis}

Data are reported as mean \pm standard deviation. Differences between the FIR-emitting outfit and placebo were assessed with a paired Student's $t$-test. A $P$ value of less than 0.05 is considered significant. All the statistical analyses were performed using SPSS computer software version 23 (IBM, Chicago, IL, USA).

\section{Results}

Twenty healthy subjects (9 women and 11 men) aged between 27 and 57 years (mean $38 \pm 12$ ) were enrolled in the study. Mean body mass index, weight and height were, respectively, $\quad 24.6 \pm 2.8 \mathrm{~kg} / \mathrm{m}^{2}, \quad 74.4 \pm 11.2 \mathrm{~kg}$ and $173.6 \pm 9.5 \mathrm{~cm}$. Non-significant differences were observed between placebo and the FIR-emitting outfit as regards CPET parameters at rest.

All subjects performed two maximal tests, ${ }^{18}$ both when wearing functionalised and placebo outfits (RER $1.14 \pm 0.1$ and $1.14 \pm 0.1$, respectively; $P=\mathrm{NS}$ ). On the FIR-emitting outfit, maximal exercise capacity was on average significantly higher than on placebo garments as shown by higher peak $\mathrm{VO}_{2}$ and endurance time (ET) (Table 1 and Figure 1). In particular, peak $\mathrm{VO}_{2}$ and ET increased in 16 and 12 cases, respectively. In spite of the limitations imposed by the exiguity of the sample, however, neither peak $\mathrm{VO}_{2}$ and ET obtained with the placebo outfit, nor volunteers' baseline characteristics could identify subjects unable to improve performance with FIR-emitting outfits. Mean $\mathrm{VO}_{2}{ }^{12,28}$ increased from $5.3 \pm 1.3 \mathrm{ml} / \mathrm{kg} / \mathrm{min}$ at rest to $38.0 \pm 8.9 \mathrm{ml} / \mathrm{kg} / \mathrm{min}$ at peak of exercise, which
Table I. Cardiopulmonary exercise test parameters during exercise.

\begin{tabular}{|c|c|c|c|}
\hline & $\begin{array}{l}\text { Functionalised } \\
\text { outfit }\end{array}$ & $\begin{array}{l}\text { Placebo } \\
\text { outfit }\end{array}$ & $P$ value \\
\hline Peak $\mathrm{VO}_{2}(\mathrm{ml} / \mathrm{kg} / \mathrm{min})$ & $38.0 \pm 8.9$ & $36.2 \pm 8.5$ & 0.006 \\
\hline Peak $\mathrm{VO}_{2}(\%)$ & $116.4 \pm 24.5$ & $111.0 \pm 24.0$ & 0.005 \\
\hline Peak $\mathrm{VCO}_{2}(\mathrm{I} / \mathrm{min})$ & $3.3 \pm 1.0$ & $3.1 \pm 1.0$ & 0.006 \\
\hline Peak HR (bpm) & $|7| \pm I \mid$ & $169 \pm 11$ & 0.015 \\
\hline Peak $\mathrm{O}_{2}$ pulse (ml/beat) & $16.6 \pm 4.7$ & $16.0 \pm 4.4$ & 0.020 \\
\hline ET $(s)$ & $592 \pm 85$ & $570 \pm 71$ & 0.009 \\
\hline AT $\mathrm{VO}_{2}(\%)$ & $72.8 \pm 19.3$ & $64.4 \pm 13.9$ & 0.003 \\
\hline AT $\mathrm{VO}_{2}(\mathrm{ml} / \mathrm{kg} / \mathrm{min})$ & $23.5 \pm 6.4$ & $20.9 \pm 5.4$ & 0.005 \\
\hline AT $\mathrm{O}_{2}$ pulse (ml/beat) & $13.3 \pm 3.8$ & $12.4 \pm 3.3$ & 0.030 \\
\hline AT time $(\mathrm{s})$ & $461 \pm 93$ & $417 \pm 103$ & 0.001 \\
\hline AT/ET ratio (\%) & $79 \pm 16$ & $73 \pm 18$ & 0.031 \\
\hline AT $\mathrm{VCO}_{2}(\mathrm{l} / \mathrm{min})$ & $1.6 \pm 0.6$ & $\mathrm{I} .4 \pm 0.5$ & 0.006 \\
\hline AT HR (bpm) & $132 \pm 16$ & $126 \pm 15$ & 0.001 \\
\hline
\end{tabular}

Peak $\mathrm{VO}_{2}$ : oxygen uptake at peak of exercise; Peak $\mathrm{VO}_{2}$ (\%): oxygen uptake at peak of exercise, expressed as percentage of predicted maximal oxygen uptake; Peak $\mathrm{VCO}_{2}$ : carbon dioxide production at peak of exercise; Peak HR: heart rate at peak of exercise; Peak $\mathrm{O}_{2}$ pulse: oxygen pulse at peak of exercise; ET: endurance time; $\mathrm{AT} \mathrm{VO}_{2}$ : oxygen uptake at the anaerobic threshold; $\mathrm{AT} \mathrm{VO}_{2}$ (\%): oxygen uptake at the anaerobic threshold, expressed as percentage of predicted maximal oxygen uptake; AT $\mathrm{O}_{2}$ pulse: oxygen pulse at the anaerobic threshold; AT time: time of exercise at the anaerobic threshold; AT/ET ratio: ratio between time of exercise at the anaerobic threshold and endurance time; AT $\mathrm{VCO}_{2}$ : carbon dioxide production at the anaerobic threshold; AT HR: heart rate at the anaerobic threshold.

corresponds to $116.4 \pm 24.5 \%$ of predicted maximal $\mathrm{VO}_{2}$, with FIR-emitting outfits, and from $5.3 \pm 1.4 \mathrm{ml} /$ $\mathrm{kg} / \mathrm{min}$ at rest to $36.2 \pm 8.5 \mathrm{ml} / \mathrm{kg} / \mathrm{min}$ at peak, which is $111.0 \pm 24.0 \%$ of predicted maximal $\mathrm{VO}_{2}$ with the placebo garments. Accordingly, peak $\mathrm{VCO}_{2}$, peak HR and peak $\mathrm{O}_{2}$ pulse $^{18}$ were significantly higher with functionalised outfits (Table 1).

The AT was significantly delayed with bioceramics, as demonstrated by longer AT time and AT/ET ratio with FIR-emitting garments in comparison to placebo; furthermore, AT $\mathrm{VO}_{2}, \mathrm{AT} \mathrm{O}_{2}$ pulse and consequently AT $\mathrm{VCO}_{2}$ and AT HR were significantly higher in functionalised versus placebo outfits (Table 1).

No significant differences were observed for ventilation $^{18}$ at peak of exercise $(93.1 \pm 26.11 / \mathrm{min}$ with the FIR-emitting outfit vs. $88.5 \pm 19.61 /$ min with placebo; $P=0.156)$.

Finally, BLC did not differ significantly between the two garments until the eighth minute of exercise, while afterwards a significantly higher lactate concentration was observed with the placebo outfit compared to functionalised garments (at 10 minutes, respectively, $4.4 \pm 0.96$ vs. $3.6 \pm 0.83 \mathrm{mmol} / \mathrm{L} ; P=0.02$; Figure 2 ). Such a significant difference of BLC between the two 


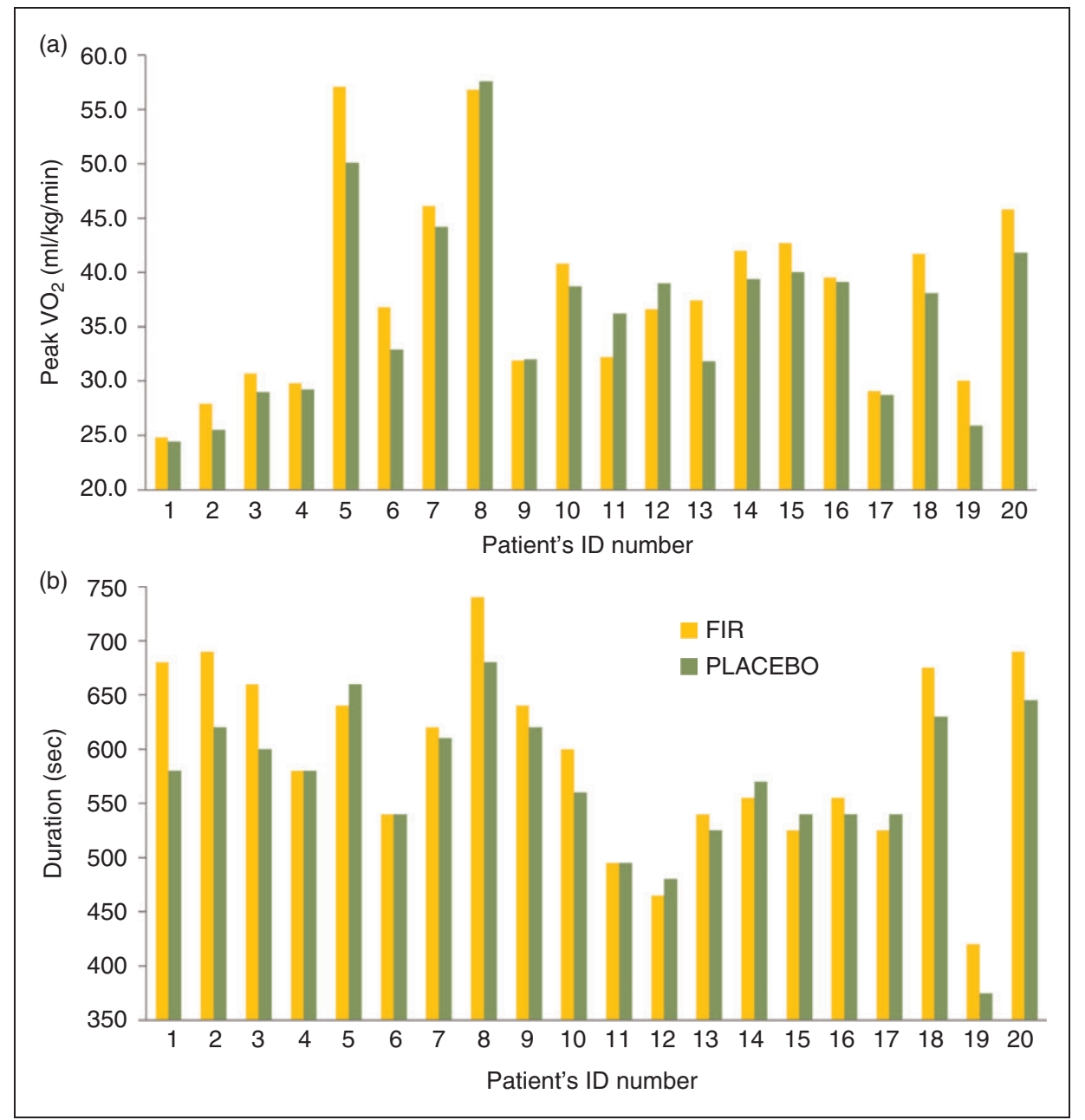

Figure I. (a) Peak oxygen uptake of each subject performing a maximal cardiopulmonary exercise test, respectively wearing functionalised and placebo garments. (b) Endurance time of each subject performing a maximal cardiopulmonary exercise test, respectively wearing functionalised and placebo garments.

Peak $\mathrm{VO}_{2}$ : oxygen uptake at peak of exercise; FIR: far-infrared radiation-emitting garments.

test conditions was correspondent to an average $20 \%$ lower BLC concentration with the FIR-emitting versus non-functionalised outfit.

From the analysis of microclimate mean temperatures during exercise, no significant differences emerged between functionalised and placebo garments $\left(29.9 \pm 1.5\right.$ vs. $30.0 \pm 0.7^{\circ} \mathrm{C}$, respectively; $\left.P=0.66\right)$.

\section{Discussion}

The main findings of the present study were as reported: active healthy subjects wearing fabrics impregnated with FIR-emitting nanoparticles (a) reached a significantly higher $\mathrm{VO}_{2}$ at submaximal (AT) and maximal (peak) efforts; (b) exercised for a longer time during maximal incremental exercise test; (c) showed a significantly delayed AT, which means that recourse to anaerobic processes to produce energy during physical activity ${ }^{24}$ came later in comparison to the same subjects wearing garments without the same properties. This is in line with different trends of BLC observed with functionalised and placebo outfits, that diverged from the eighth minute (around the AT) and reached a significant difference at 10 minutes of exercise (Figure 2). These effects could be explained by an enhanced muscular oxygen delivery thanks to the non-thermic increase in muscular perfusion induced by FIR.

As lactates start accumulating in the working muscles beyond the AT, determining the emergence of muscular fatigue, ${ }^{29,30}$ the delay in AT is probably a determinant of longer exercise observed with the FIRemitting outfit compared to placebo. Other studies on humans have demonstrated a correlation between the decline in intracellular skeletal muscle $\mathrm{pH}$ and the reduction of force production, due to the effect of 


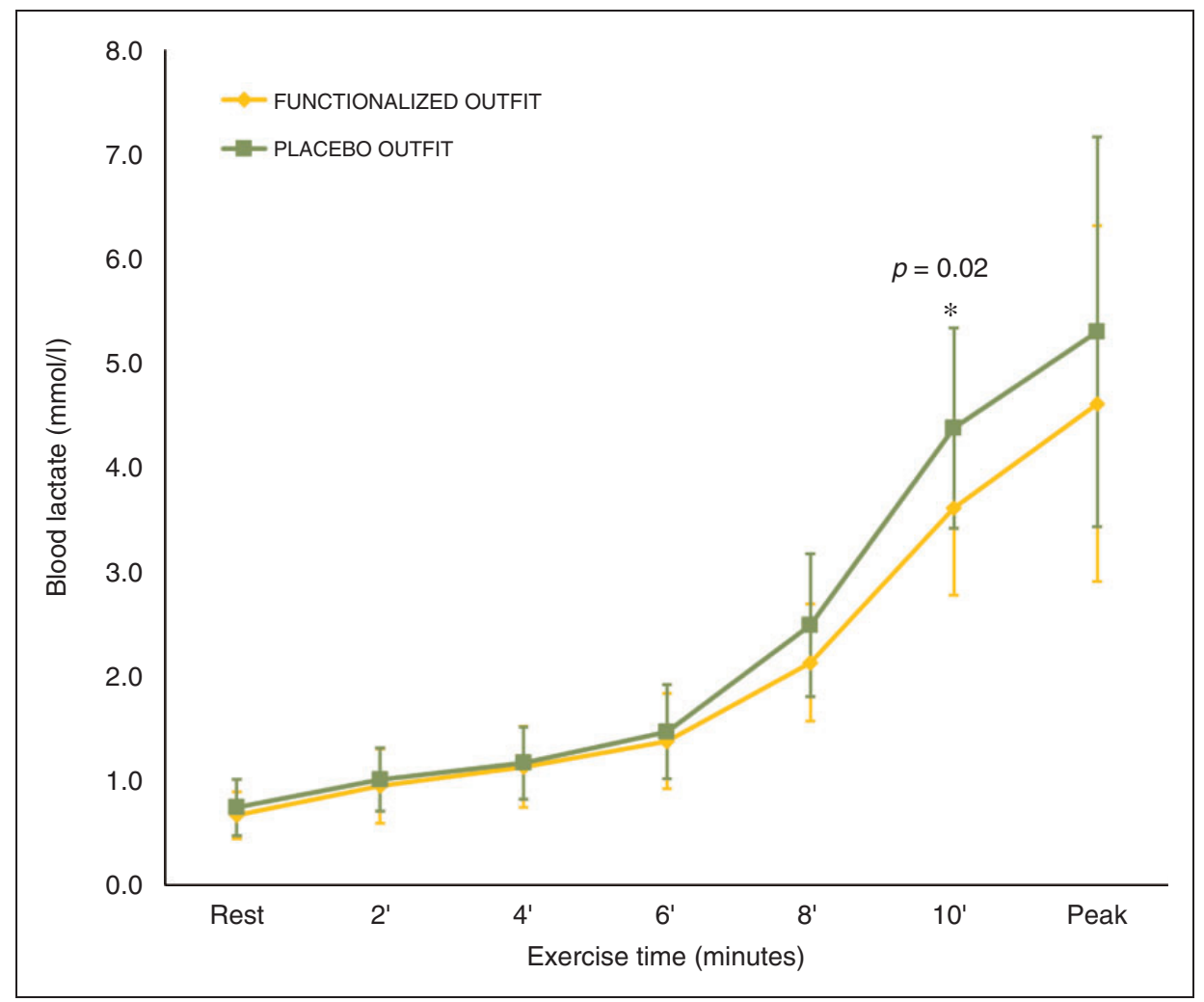

Figure 2. Mean blood lactate concentration during exercise with the two outfits, respectively. Rest: measurement at rest; Peak: measurement at peak of exercise.

acidification on myofibril proteins. ${ }^{2}$ Leung and colleagues studied in vitro effects of FIR-emitting ceramic powders on isolated amphibian gastrocnemius muscle and demonstrated that bioceramics delayed the onset of fatigue induced by repeated activity of the muscle and higher muscular $\mathrm{pH}$ in the FIR group versus controls. ${ }^{2}$

However, the exact mechanisms by which FIR irradiation exerts its biological activities and effects on exercise performance are still not clear.

In the literature, two types of FIR are described. ${ }^{2,4,8}$ One is thermic FIR (FIR sauna and FIR generators powered by electricity) that transfers heat to the human body and increases the local temperature. ${ }^{4,9}$ This would induce muscular vasodilation and enhance muscular blood flow, ${ }^{4,8}$ that could be responsible for higher oxygen peripheral delivery, ${ }^{4,12,16,25}$ enhanced evacuation of oedema and facilitated removal of metabolic wastes. ${ }^{31}$ Interestingly, in Japan FIR dry sauna to warm the human body (Waon therapy) by $1.0-1.2^{\circ} \mathrm{C}$ has commonly been used to treat heart failure patients, even in an advanced state, and is indicated as class I for these patients in the Japanese heart failure guidelines. ${ }^{32}$ In fact, Waon therapy has been shown to reduce preload and afterload through peripheral vasodilation, to enhance nitric oxide synthase (NOS), to improve autonomic nervous activity ${ }^{32-34}$ and to reduce oxidative stress. Vasodilation induced by the overexpression of NOS and reduced sympathetic nervous activity would increase cardiac output and reduce pulmonary congestion. All these biological effects have been demonstrated to improve heart failure symptoms, exercise tolerance and quality of life when Waon therapy is applied on top of optimal medical therapy. ${ }^{32}$

However, garments furnished by CNILIT $^{\circledR}$ have exclusively non-thermic biological properties, as confirmed by the analysis of microclimate temperatures and in line with what the literature reports about fabrics enriched with bioceramics. ${ }^{4}$ This type of FIR emits such low irradiance that tissue is not heated when irradiated. ${ }^{1,3,4,7}$ However, similarly to heating FIR, nonthermic FIR is associated with increased skin blood flow, presumably secondary to a microvascular dilation induced by nitric oxide (NO). ${ }^{1,3,4,7}$ This would be produced in endothelial cells $\mathrm{s}^{7,8}$ by NOS, both because of a FIR-induced upregulated expression of $\operatorname{NOS}^{8}$ and because of an increased level of calmodulin with secondary calcium/calmodulin-mediated stimulation of constitutive NOS. ${ }^{3}$ Pretreatment of FIR-irradiated rat models with inhibitors of NOS prevents the enhancing effect on microcirculation. ${ }^{7}$ Calbet and colleagues ${ }^{16}$ analysed the effects of vasodilation on haemodynamics by infusion of ATP during an incremental test in 
healthy volunteers. Both systemic and leg peak $\mathrm{VO}_{2}$ were not enhanced by augmented limb perfusion, nor were relevant differences observed in venous lactate concentration, because of a significant rise in venous oxygen content. ${ }^{16}$ However, the entity of vasodilation generated by the infusion of ATP may be different from that provoked by FIR. It could be that infused ATP induces an exaggerated response in active muscles, overriding their maximal vasodilatory capacity and deviating part of the blood flow from exercising muscles to less active tissues. ${ }^{16}$ This, in turn, would determine an increase in venous oxygen content, and reduce $\Delta \mathrm{a}-\mathrm{vO}_{2}$ with unchanged $\mathrm{VO}_{2}$. Moreover, some authors, in the setting of muscular vasodilation induced by local heating of the exercising muscle, found an increase in leg blood flow proportionally correlated with limb $\mathrm{VO}_{2}$ during incremental exercise, ${ }^{14,15}$ consistent with the evidence of a significantly higher peripheral oxygen delivery ${ }^{15}$ and attenuated drop in venous $\mathrm{pH}^{14}$

In addition, besides increased muscular perfusion, small amounts of energy reflected by non-heating FIR fabrics would cause small perturbations in cellular membrane structure where several ion channels are embedded, especially in mitochondrial membranes. ${ }^{4}$ FIR-induced opening of mitochondrial ion channels, especially calcium channels, would increase mitochondrial respiration and then oxygen consumption and ATP production. ${ }^{4}$ Finally, FIR is also supposed to reduce oxidative stress, ${ }^{1-3,7,8,11}$ thus inhibiting cytotoxicity $^{1}$ and probably contributing to delay metabolic acidosis and muscular fatigue. ${ }^{1}$

Although inconclusive data reported in the literature seem to support our hypothesis that exercise in healthy subjects could be favourably influenced by non-thermic activities of garments knitted with bioceramics. In fact, functionalised sport clothes might induce local hyperaemia and enhance peripheral oxygen delivery (and removal of waste materials) in exercising limb muscles, which is the main limiting factor of maximal aerobic performance in healthy subjects after endurance training. ${ }^{35}$ The increase in oxygen delivery would delay the emergence of anaerobic metabolism and muscular fatigue, on the one hand, and increase exercise capacity on the other hand. Amelioration of functional capacity at the AT is especially intriguing, considering that most daily activities are of moderate submaximal intensity.

\section{Study limitations}

Some study limitations need to be recognised. First, we studied a short-lasting (approximately 10 minutes) exercise test, therefore our results cannot be generalised to all effort durations, i.e. to very short or more prolonged efforts, in which a different metabolism or metabolic rate are present. Data in this regard are, to our knowledge, not available and specific studies need to be done.

Second, we tested healthy fit subjects. Accordingly, our data cannot be extrapolated to patients with cardiac or pulmonary exercise limitation, to deconditioned subjects or to patients with limited vasodilating capacity, such as patients with diabetes and vascular diseases. The same applies to elite athletes.

\section{Future perspectives}

Although the efficacy and safety of FIR thermic saunas have already been demonstrated in patients with chronic heart failure, ${ }^{32}$ FIR garments have never been tested in patients. However, patients affected by chronic diseases, such as those with heart failure and pneumopathies, show a relevant compromise of exercise capacity due to an inadequate peripheral oxygen delivery and abnormal muscle function so that anaerobic sources for energy production are anticipated. ${ }^{36,37}$ Moreover, the effects of exercise training are a function of exercise intensity so that the higher the intensity, the better the results of any rehabilitation programme. ${ }^{37,38}$

Consequently, it is intriguing to hypothesise that even fabrics enriched in bioceramics could increase muscular perfusion in chronic patients during exercise. It has been demonstrated that exercise training in heart failure patients induces a significant improvement in functional capacity by increasing exercise cardiac output; ${ }^{37}$ FIR would contribute to an increase in oxygen delivery and a post-position of AT, thus enhancing the well-recognised benefits of physical rehabilitation. In addition, NO is involved in the regulation of sympathetic nervous activity in humans, therefore we could hypothesise that FIR-emitting garments could also modulate the hyperactivation of the sympathetic nervous system that typically occurs in chronic heart failure patients.

We believe that further studies are definitively needed as regards safety, efficacy, effects on symptoms and timing of FIR garments in chronic patients; investigations should also be encouraged on the possible effects of such rehabilitation programmes on exercise capacity (whether submaximal or maximal) and prognosis of chronic patients, as evaluated by CPET parameters. ${ }^{39,40}$

\section{Conclusions}

In conclusion, the use of FIR-emitting garments generated by the incorporation of natural minerals is associated with an improvement in submaximal and maximal exercise capacity and a delay of AT in active healthy subjects, presumably by an increase in oxygen peripheral delivery. This might have implications for 
patients; therefore, further testing of FIR-emitting garments in chronic patients engaged in rehabilitation programmes is justified.

\section{Author contribution}

$\mathrm{AP}, \mathrm{BM}$ and $\mathrm{BP}$ contributed to the conception and/or design of the study. MV, CM, BM, FA, DF and AP contributed to the acquisition, analysis, or interpretation of data for the work. MV, CM and AP drafted the manuscript. All authors critically revised the manuscript, gave final approval and agree to be accountable for all aspects of the work ensuring integrity and accuracy.

\section{Acknowledgements}

The authors would like to thank Angela Galantucci for her relevant nursing help.

\section{Declaration of conflicting interests}

The authors declared the following potential conflicts of interest with respect to the research, authorship, and/or publication of this article: ${ }^{C N I L I T}{ }^{\circledR}$ Ltd. is the owner of far-infrared emitting garments. They are responsible for the results of the study tests performed on 10 volunteers and they provided the outfits for the tests performed in the exercise laboratory at Centro Cardiologico Monzino. Centro Cardiologico Monzino did not receive any financial support from CNILIT $^{\circledR}$.

Valentina Mantegazza, Mauro Contini, Ada Ferri, Francesca Dotti and Piergiuseppe Agostoni declare that they have no conflicts of interest.

Pierluigi Berardi received personal fees from ${ }^{C}$ NILIT $^{\circledR}$ for employment.

Maurizia Botti received personal fees from $\mathbb{C N I L I T ~}^{\circledR}$ for expert consulting.

\section{Funding}

The authors disclosed receipt of the following financial support for the research, authorship, and/or publication of this article: this research was supported by Italian Ministry of Health and ${ }^{C}$ NILIT $^{\mathbb{B}}$ Ltd. provided the outfits for the tests.

\section{References}

1. Leung TK. In vitro and in vivo studies of the biological effects of bioceramic (a material of emitting high performance far-infrared ray) irradiation. Chinese J Physiol 2015; 58: $147-155$.

2. Leung TK, Lee CM, Tsai SY, et al. A pilot study of ceramic powder far-infrared ray irradiation (cFIR) on physiology: observation of cell cultures and amphibian skeletal muscle. Chinese J Physiol 2011; 54: 247-254.

3. Leung TK, Kuo CH, Lee CM, et al. Physiological effects of bioceramic material: harvard step, resting metabolic rate and treadmill running assessments. Chinese J Physiol 2013; 56: 334-340.

4. Vatansever $F$ and Hamblin MR. Far infrared radiation (FIR): its biological effects and medical applications. Photon Lasers Med 2012; 4: 255-266.
5. Anderson DM, Fessler JR, Pooley MA, et al. Infrared radiative properties and thermal modeling of ceramicembedded textile fabrics. Biomed Optics Express 2017; 8: 1698-1711.

6. Pooley MA, Anderson DM, Beckham HW, et al. Engineered emissivity of textile fabrics by the inclusion of ceramic particles. Optics Express 2016; 24: 10556-10564.

7. Yu SY, Chiu JH, Yang SD, et al. Biological effect of farinfrared therapy on increasing skin microcirculation in rats. Photodermatol, Photoimmunol Photomed 2006; 22: 78-86.

8. Lin CC, Chang CF, Lai MY, et al. Far-infrared therapy: a novel treatment to improve access blood flow and unassisted patency of arteriovenous fistula in hemodialysis patients. J Am Soc Nephrol: JASN 2007; 18: 985-992.

9. Inoue $\mathrm{S}$ and Kabaya $\mathrm{M}$. Biological activities caused by far-infrared radiation. Int J Biometeorol 1989; 33: $145-150$.

10. Shui S, Wang X, Chiang JY, et al. Far-infrared therapy for cardiovascular, autoimmune, and other chronic health problems: a systematic review. Exp Biol Med (Maywood) 2015; 240: 1257-1265.

11. Zhang R, Mio Y, Pratt PF, et al. Near infrared light protects cardiomyocytes from hypoxia and reoxygenation injury by a nitric oxide dependent mechanism. $\mathrm{J} \mathrm{Mol} \mathrm{Cell}$ Cardiol 2009; 46: 4-14.

12. Agostoni PG, Bussotti M, Nani F, et al. Oxygen consumption. Cardiologia 1999; 44: 987-992.

13. Pearson J, Low DA, Stohr E, et al. Hemodynamic responses to heat stress in the resting and exercising human leg: insight into the effect of temperature on skeletal muscle blood flow. Am J Physiol Regul, Integr Comp Physiol 2011; 300: R663-R673.

14. Gonzalez-Alonso J, Calbet JA, Boushel R, et al. Blood temperature and perfusion to exercising and non-exercising human limbs. Exp Physiol 2015; 100: 1118-1131.

15. Chiesa ST, Trangmar SJ, Kalsi KK, et al. Local temperature-sensitive mechanisms are important mediators of limb tissue hyperemia in the heat-stressed human at rest and during small muscle mass exercise. Am J Physiol Heart Circ Physiol 2015; 309: H369-H380.

16. Calbet JA, Lundby C, Sander M, et al. Effects of ATPinduced leg vasodilation on $\mathrm{VO}_{2}$ peak and leg $\mathrm{O}_{2}$ extraction during maximal exercise in humans. Am J Physiol Regul, Integr Comp Physiol 2006; 291: R447-R453.

17. Koch B, Schaper C, Ittermann T, et al. Reference values for cardiopulmonary exercise testing in healthy volunteers: the SHIP study. Eur Respir J 2009; 33: 389-397.

18. Balady GJ, Arena R, Sietsema K, et al. Clinician's guide to cardiopulmonary exercise testing in adults: a scientific statement from the American Heart Association. Circulation 2010; 122: 191-225.

19. Palange P, Schena F and Wasserman K. Il Test da sforzo cardiopolmonare. Teoria e applicazioni, I ed. Pavona di Albano: COSMED, 2001.

20. Myers J, Arena R, Franklin B, et al. Recommendations for clinical exercise laboratories: a scientific statement from the american heart association. Circulation 2009; 119: 3144-3161. 
21. Mezzani A. Cardiopulmonary exercise testing: basics of methodology and measurements. Ann Am Thor Soc 2017; 14: S3-S11.

22. Wasserman K, Hansen JE, Sue DY, et al. Principles of exercise testing and interpretation, 5th edn. Philadelphia: Lea \& Febiger, 2011.

23. Sullivan MJ and Cobb FR. The anaerobic threshold in chronic heart failure. Relation to blood lactate, ventilatory basis, reproducibility, and response to exercise training. Circulation 1990; 81: II47-II58.

24. Wasserman K, Beaver WL and Whipp BJ. Gas exchange theory and the lactic acidosis (anaerobic) threshold. Circulation 1990; 81: II14-II30.

25. Piepoli MF, Corra U, Agostoni PG, et al. Statement on cardiopulmonary exercise testing in chronic heart failure due to left ventricular dysfunction: recommendations for performance and interpretation. Part I: definition of cardiopulmonary exercise testing parameters for appropriate use in chronic heart failure. Eur J Cardiovasc Prev Rehabil: Official journal of the European Society of Cardiology, Working Groups on Epidemiology and Prevention and Cardiac Rehabilitation and Exercise Physiology 2006; 13: 150-164.

26. Solberg G, Robstad B, Skjonsberg OH, et al. Respiratory gas exchange indices for estimating the anaerobic threshold. J Sports Sci Med 2005; 4: 29-36.

27. Wasserman K, Stringer WW, Casaburi R, et al. Determination of the anaerobic threshold by gas exchange: biochemical considerations, methodology and physiological effects. Zeitschrift fur Kardiologie 1994; 83(Suppl. 3): 1-12.

28. Mezzani A, Agostoni P, Cohen-Solal A, et al. Standards for the use of cardiopulmonary exercise testing for the functional evaluation of cardiac patients: a report from the Exercise Physiology Section of the European Association for Cardiovascular Prevention and Rehabilitation. Eur J Cardiovasc Prev Rehabil: Official journal of the European Society of Cardiology, Working Groups on Epidemiology and Prevention and Cardiac Rehabilitation and Exercise Physiology 2009; 16: 249-267.

29. Wasserman K. Determinants and detection of anaerobic threshold and consequences of exercise above it. Circulation 1987; 76: VI29-VI39.
30. Wasserman $\mathrm{K}$ and Koike A. Is the anaerobic threshold truly anaerobic? Chest 1992; 101: 211S-218S.

31. Hausswirth C, Louis J, Bieuzen F, et al. Effects of wholebody cryotherapy vs. far-infrared vs. passive modalities on recovery from exercise-induced muscle damage in highly-trained runners. PloS One 2011; 6: e27749.

32. Tei C, Imamura $\mathrm{T}$, Kinugawa $\mathrm{K}$, et al. Waon therapy for managing chronic heart failure - results from a Multicenter Prospective Randomized WAON-CHF Study. Circulation J: Official journal of the Japanese Circulation Society 2016; 80: 827-834.

33. Kuwahata S, Miyata M, Fujita S, et al. Improvement of autonomic nervous activity by Waon therapy in patients with chronic heart failure. J Cardiol 2011; 57: 100-106.

34. Munemoto T, Soejima Y, Masuda A, et al. Increase in the Regional Cerebral Blood Flow Following Waon Therapy in Patients with Chronic Fatigue Syndrome: a pilot study. Intern Med 2017; 56: 1817-1824.

35. Mezzani A and Guazzi M. Balancing the evidence on the cardiovascular determinants of oxygen uptake improvement after endurance training in the elderly: what are the next steps? Eur J Prev Cardiol 2016; 23: 730-732.

36. Kisaka T, Stringer WW, Koike A, et al. Mechanisms that modulate peripheral oxygen delivery during exercise in heart failure. Ann Am Thor Soc 2017; 14: S40-S47.

37. Cattadori G, Schmid JP, Brugger N, et al. Hemodynamic effects of exercise training in heart failure. J Cardiac Fail 2011; 17: 916-922.

38. Ponikowski P, Voors AA, Anker SD, et al. 2016 ESC Guidelines for the diagnosis and treatment of acute and chronic heart failure: the Task Force for the Diagnosis and Treatment of Acute and Chronic Heart Failure of the European Society of Cardiology (ESC). Developed with the special contribution of the Heart Failure Association (HFA) of the ESC. Eur J Heart Fail 2016; 18: 891-975.

39. Sato T, Yoshihisa A, Kanno Y, et al. Cardiopulmonary exercise testing as prognostic indicators: comparisons among heart failure patients with reduced, mid-range and preserved ejection fraction. Eur J Prev Cardiol 2017; 24: 1979-1987.

40. Bongers BC, Hulzebos EH, Helbing WA, et al. Response profiles of oxygen uptake efficiency during exercise in healthy children. Eur J Prev Cardiol 2016; 23: $865-873$. 